\title{
Comparison of clinical outcomes and safety of catheter ablation for atrial fibrillation supported by data from CT scan or three-dimensional rotational angiogram of left atrium and pulmonary veins
}

\author{
Frantisek Lehar, Zdenek Starek, Jiri Jez, Miroslav Novak, Jiri Wolf, Radka Stepanova, \\ Peter Kruzliak, Tomas Kulik, Alena Zbankova, Radek Jancar, Jiri Vitovec
}

\begin{abstract}
Background. Catheter ablation in the left atrium has become a common therapeutic strategy in the management of atrial fibrillation (AF). The high degree of success and safety profile of this procedure is dependent on precise knowledge of the true anatomy in the chamber. This information is imported mostly from cardiac computed tomography. A novel method for imaging the left atrial anatomy is three-dimensional rotational angiography (3DRA).

Methods. The aim of our study was to the compare clinical outcome and safety of catheter ablation for atrial fibrillation guided by 3DRA vs. conventional CT scan. One hundred and twenty-five patients referred for AF catheter ablation at St. Anne's University Hospital Brno were included in the retrospective analysis of clinical outcome within the first year after the procedure.
\end{abstract}

Results. There was a close correlation in overall procedural parameters between the groups. The frequency of recurrent episodes of AF (24\% in CT-guided group vs. $27 \%$ in 3DRA-guided group, $P=0.721$ ) as well as the onset of atypical atrial flutter after the procedure ( $10 \%$ vs. $8 \%$, respectively, $P=0.731)$ were similar in both groups. No difference in the number of patients necessitating repeat ablation ( $5 \%$ vs. $5 \%, P=0.984$ ) was found. Procedural complications of ablations guided by 3DRA were comparable with those guided by CT ( $2 \%$ vs. $3 \%$, respectively, $P=0.568$ ).

Conclusion. 3DRA has proven to be a safe and simple method for imaging the left atrium and guiding catheter ablation for AF. This approach is anticipated to become a new standard in 3D reconstruction of the left atrium.

Keywords: atrial fibrillation, catheter ablation, electrophysiology, three dimensional rotational atriography, computed tomography, imaging, left atrium

Received: October 14, 2013; Accepted: July 3, 2014; Available online July 17, 2014

http://dx.doi.org/10.5507/bp.2014.040

International Clinical Research Center, Department of Cardiovascular Disease, St. Anne's University Hospital in Brno and Faculty of Medicine, Masaryk University Brno, Czech Republic

Corresponding author: Frantisek Lehar, e-mail: frantisek.lehar@fnusa.cz

\section{INTRODUCTION}

Catheter ablation for atrial fibrillation is a technically challenging but highly effective left atrial procedure ${ }^{1}$. Given the variability of the left atrium and pulmonary vein ostia, precise knowledge of true anatomy is the key to a successful and safe intervention ${ }^{2}$. Therefore, preprocedural computer tomography (CT) or magnetic resonance (MR) is frequently used as anatomical guidance for catheter ablation ${ }^{3-5}$. A novel method allowing reconstruction of the left atrium is three-dimensional rotational angiogram (3DRA), which is carried out intra-procedurally right in the EP $1 \mathrm{ab}^{6,7}$. The acquired $3 \mathrm{D}$ volume is then used as a template for non-fluoroscopic 3D electroanatomic mapping in the systems Carto (Biosense Webster, Diamond Bar, CA, USA) or Velocity (St. Jude Medical, St. Paul, MN, USA) (ref. ${ }^{8,9}$ ). Three-dimensional rotational angiogram also enables merging the resulting $3 \mathrm{D}$ image with live fluoroscopy, which is a great help for orientation in the left atrium during the map acquisition (Fig. 1). Moreover, the anatomical data obtained from 3D rotational angiogram of left atrium has proven to be compa- rable with the information from conventional CT scan and radiation exposure is lower by using 3DRA (ref. ${ }^{10,11}$ ). Another advantage is the possibility of integrating the 3D image from the rotational angiography into the electroanatomical mapping system, which facilitates the execution of AF ablation and reduces the total procedural time and radiation exposure while providing similar clinical outcomes $^{12}$. Previous studies have proven that the fusion of CT or MR image of the left atrium with the electroanatomical map in the Carto-Merge system is beneficial for the clinical outcome of the AF ablation and reduction in fluoroscopic guidance ${ }^{4,13,14}$. The range of 3D rotational angiography application is even wider. There are reports of registration of $3 \mathrm{D}$ volume obtained from 3DRA with intracardiac echocardiography (ICE) allowing for electroanatomical mapping of the left atrium. This was the first ever image integration of two left atrial reconstructions by means of two different intraprocedural methods of noninvasive cardiac imaging - 3D rotational angiography and ICE-based image registration in electroanatomical mapping system ${ }^{15}$.

Three-dimensional rotational angiogram is performed 


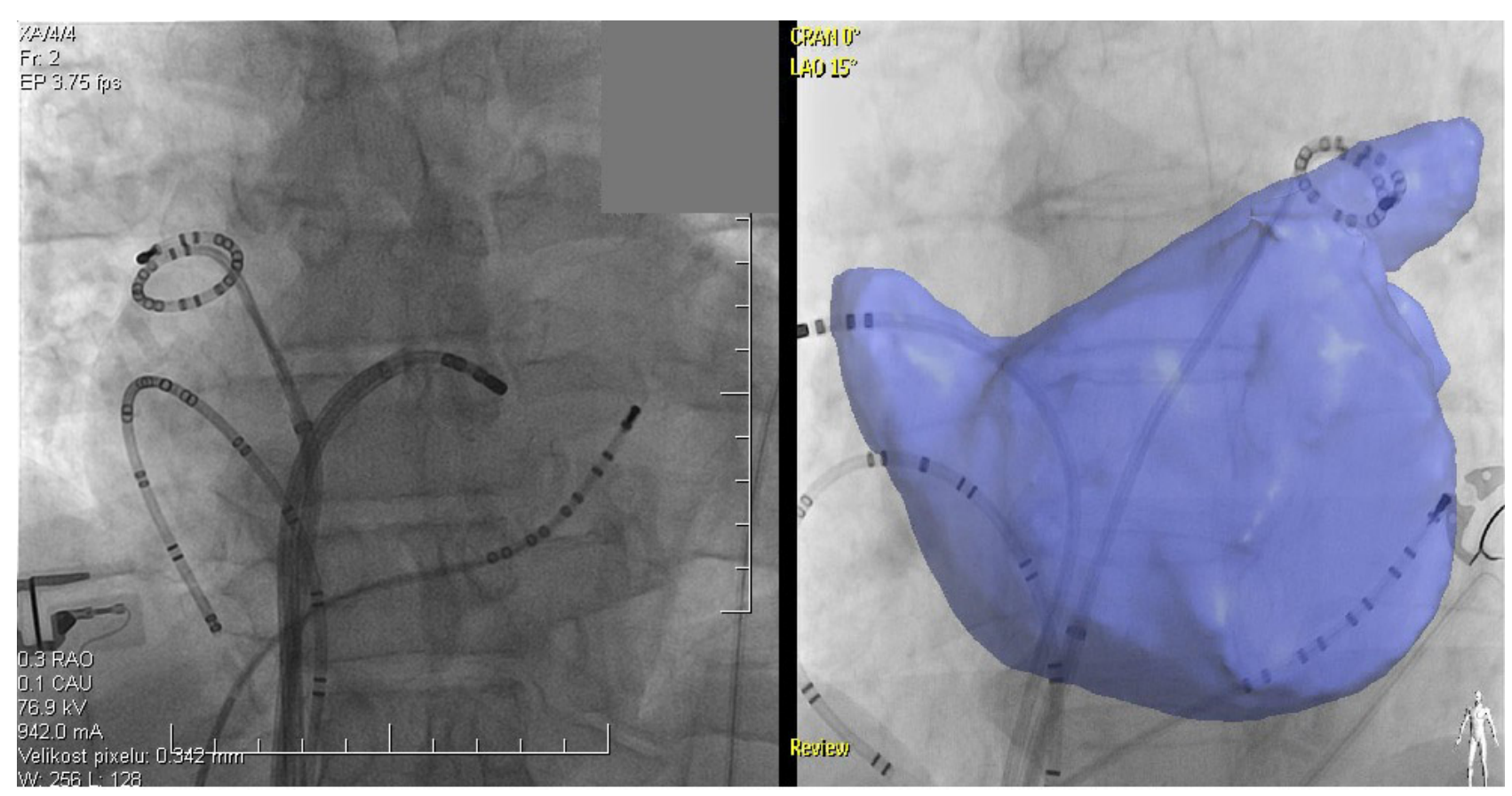

Fig. 1. On the left, there is a skiascopic view of catheter ablation of atrial fibrillation without using three-dimensional rotational angiography. On the right, there is a fused image of the skiascopic view and the $3 \mathrm{D}$ image of the left atrium obtained from three dimensional angiography. This fused image is great guidance for catheter ablation of atrial fibrillation.

using cardiac X-ray system with custom software for 3D image post-processing. Following the initial intracardial contrast injection, a fixed C-arm rotates around the patient and acquires a series of $\mathrm{x}$-ray images immediately reconstructed by a software algorithm into the $3 \mathrm{D}$ volume representing the left atrium and pulmonary vein ostia. The contrast medium can be injected either directly into the left atrium with subsequent rapid ventricular pacing or intravenous administration of adenosine to induce shortterm asystoly and allow for homogenous contrast opacification, or indirectly in the right atrium with a delay of 9 s preceding the initiation of rotational run to permit the transit of contrast medium through the lungs into the left atrium $^{16,17}$. Advanced angiography systems equipped with 3DRA (e.g. EP Navigator - Philips, DynaCT Cardiac Siemens, Innova - GE Healthcare) are currently available to enhance and support electrophysiological intervention.

From 2010 to June 2013, our center performed more than 408 left atrial and 33 right or left ventricular angiograms. To the best of our knowledge, there are no data available on the clinical outcome of catheter ablation for atrial fibrillation guided by $3 \mathrm{D}$ rotational angiogram of left atrium. The aim of our study was to compare clinical outcome and safety of the catheter ablation for atrial fibrillation guided by 3D rotational angiography vs. conventional CT scan.

\section{METHODS}

One hundred and twenty-five patients referred for AF catheter ablation at St. Anne's University Hospital in Brno were included in the retrospective analysis of clinical out- come within the first year after the procedure. Data from January 2011 to August 2011 reported only CT-guided ablations while the majority of the procedures between September 2011 and August 2012 have already used the $3 \mathrm{D}$ rotational angiography with left atrial injection and those were involved in the study. Had there been a history of iodine allergy or renal insufficiency indicated by a decline in glomerular filtration rate of less than $45 \mathrm{~mL} /$ min, no 3DRA or CT were performed.

\section{Patient preparation}

All patients underwent a full clinical assessment in an outpatient service for cardiac arrhythmia and were submitted to catheter ablation for drug refractory paroxysmal or persistent atrial fibrillation. Transthoracic echocardiography was performed preoperatively in all of them. If considered necessary, anticoagulant therapy followed CHA2DS2-VASc score guidelines and was discontinued five days prior to the scheduled procedure ${ }^{18}$. Patients were supplemented with subcutaneous low molecular heparin adjusted according to their weight until the day of the procedure to compensate for ineffective levels of INR. Those treated from January 2011 to August 2011 underwent preprocedural cardiac CT. Before the procedure, transesophageal echocardiography was performed to exclude the presence of intracardiac thrombus.

\section{Computed tomography}

Patients underwent a CT scan within seven to 14 days preceding the procedure using 64 Slice CT Scanner (GE Lightspeed VCT, General Electric, Fairfield, USA) with configuration $120 \mathrm{KV}, 800 \mathrm{mAs}$, a collimation width of $63 \times 0.625 \mathrm{~mm}$, and a spiral pitch factor of 0.98 . The 
images were then reconstructed in a resolution of $512 \mathrm{x}$ 512 pixels. Contrast medium injection $(100-150 \mathrm{~mL}$ of Ultravist 370, Bayer Pharma AG, Berlin, Germany or Iomeron 400, PNG Gerolymatos A.E.B.E., Kryoneri Athens, Greece) was administered via the peripheral vein. During the acquisition, patients remained lying down with both arms raised and breath-holding. The obtained x-ray images were then transferred to a data CD. At the time of the procedure, 3D representation of the left atrium was reconstructed in the $3 \mathrm{D}$ electroanatomical mapping system (EnSite Velocity, St. Jude Medical, St. Paul, MN, USA) based on the anatomic shell imported from CT.

\section{Three-dimensional rotation angiography}

Rotational angiography was performed using a C-arm angiography system Philips Allura Xper FD 10 with a 10-inch flat detector (Philips Healthcare, Best, The Netherlands). After injecting the contrast medium into the left atrium, $\mathrm{C}$-arm was rotated from $120^{\circ}$ right anterior oblique (RAO) to $120^{\circ}$ left anterior oblique position (LAO) over $4.1 \mathrm{~s}$. Images acquired at a rate of 30 frames per minute were then transferred to a working station and reconstructed into a $3 \mathrm{D}$ volume using the specialized software for automated segmentation (EP Navigator 3.0, Philips Healthcare, Best, The Netherlands). For a contrast injection, a pigtail catheter (Cordis, Miami, FL, USA) advanced to the left atrium was used and a bolus of 60 mL contrast medium (Ultravist $370 \mathrm{I} / \mathrm{mL}$, Bayer Pharma AG, Berlin, Germany) was administered at a speed of 15 $\mathrm{mL} / \mathrm{s}$ via power injector (Mark-V ProVis, Medrad, Inc., Indianola, PA, USA). Patients were lying down with the hands along the body and were asked for shallow breathing. Prior to contrast application, a quadrupolar steerable catheter (Irvine Biomedicals, Irvine, CA, USA) was positioned into the right atrial apex and rapid ventricular pacing of 220 beats per min was performed with a substantial decrease in blood pressure detected by the absence of oxygen saturation curve on the pulse oximetry monitor screen (Philips IntelliVue MP-20, Philips, Eindhoven, The Netherlands) (ref. ${ }^{19}$ ). Finally, the contrast medium was injected and C-arm rotation initiated with a $2 \mathrm{~s}$ delay. Along with the left atrial rotational angiography, all patients underwent a $3 \mathrm{D}$ rotational esophagography to visualize the position of the esophagus and its relation to other cardiac structures ${ }^{20}$. Opacification was achieved by swallowing 30-50 mL of barium sulfate esophageal cream (Micropaque - Guerbet, Roissy, France) five seconds before the initiation of rotational run.

\section{Catheter ablation}

All patients underwent the ablation procedure under a standard protocol using Philips Allura Xper FD10 system (Philips Healthcare, Best, The Netherlands). Sheaths and electrophysiological catheters were inserted via both femoral veins under local anaesthesia. The $8 \mathrm{~F}$ sheath was introduced through the left femoral vein and used for advancement of a decapolar catheter into the coronary sinus. Double transseptal puncture was performed via right femoral venous access with the guidance of fluoroscopy, local injection of an iodinated contrast medium with an invasive blood pressure monitoring system on a needle tip, and intracardial ultrasound. Then, both an $8.5 \mathrm{~F}$ Agilis steerable introducer and an 8F SL1 sheath (St. Jude Medical, St. Paul, MN, USA) were positioned into the left atrium and a bolus of intravenous heparin with continuous infusion adjusted according to the ACT (target levels of 350 to $300 \mathrm{~s}$ ) was administered. An irrigatedtip ablation catheter (Celsius ${ }^{\mathrm{TM}}$ Thermo-cool, Biosense Webster, Diamond Bar, CA, USA) was inserted over the Agilis steerable sheath and placed into the left atrium as well as a duodecapolar spiral catheter (ReflexionSpiral Variable Radius Catheter ${ }^{\mathrm{TM}}$, St. Jude Medical, St. Paul, MN, USA) advanced over the SL1 transseptal sheath. An electroanatomical map of the left atrium and left pulmonary veins was made based on a 3D anatomic shell from either CT or 3DRA in the EnSite NavX system
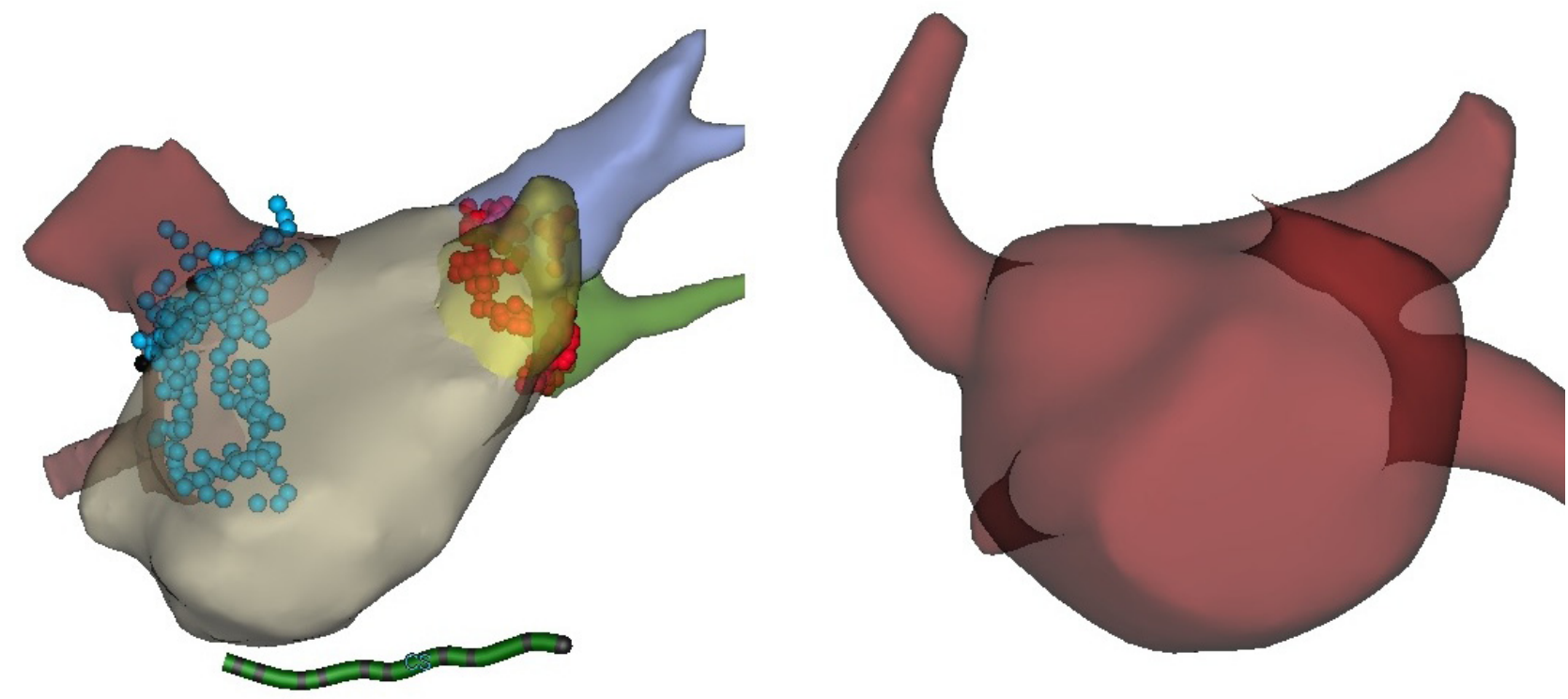

Fig. 2. On the right, there is a 3D image of the left atrium obtained from CT scan. On the left, there is shown an electroanatomical map of the left atrium based on the image obtained from CT (the red and blue points mark location of RF ablations). 

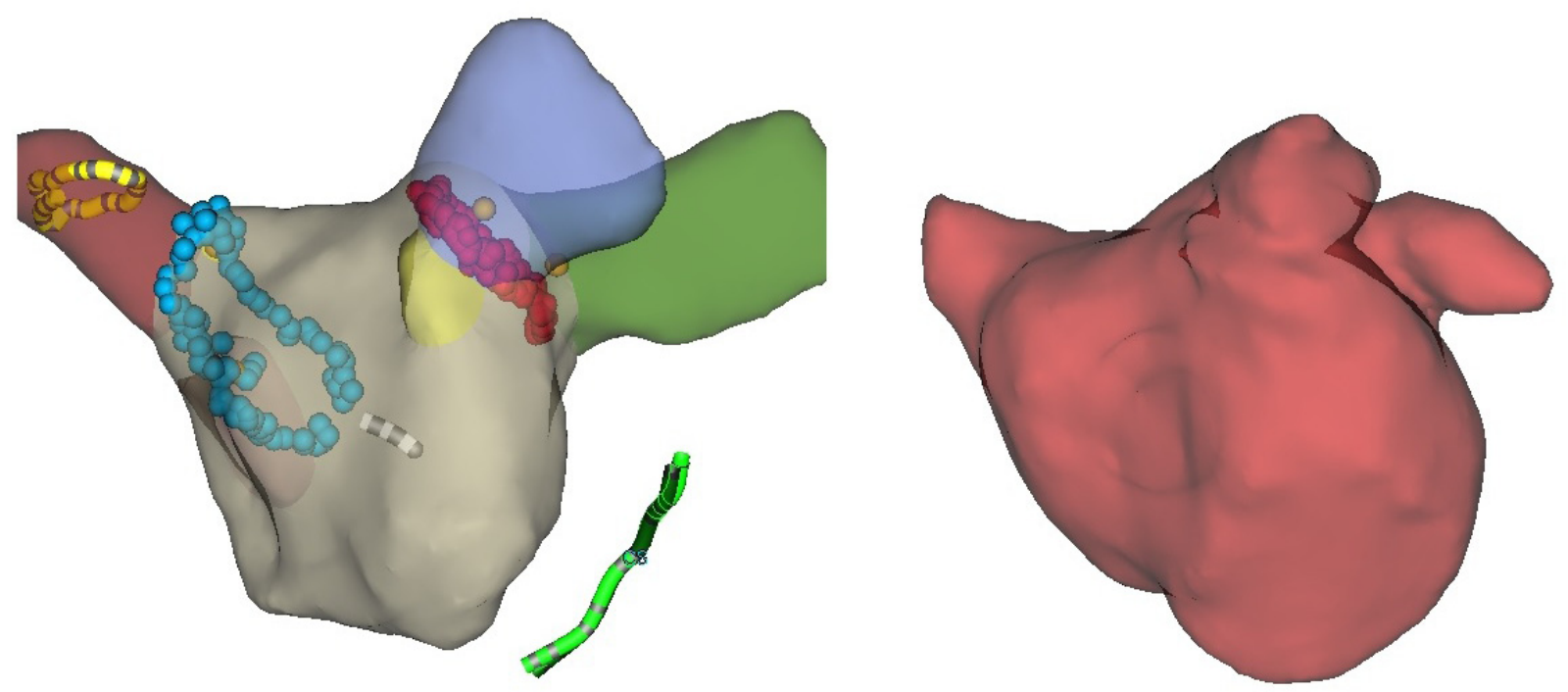

Fig. 3. On the right, there is a 3D image of the left atrium obtained from three-dimensional rotational angiography. On the left, there is shown an electroanatomical map of the left atrium based on the image obtained from CT (the red and blue points mark location of RF ablations).

(St. Jude Medical, St. Paul, MN, USA) (Fig. 2 and 3). Radio-frequency energy was delivered creating circular ablation lesions to isolate pulmonary vein ostia. The successful isolation was defined by achieving the entrance and/or exit block. In patients with persistent atrial fibrillation, adjacent lines of ablation at the left atrial roof, mitral isthmus and distal coronary sinus were performed. During the procedure, patients remained under mild sedation with midazolam and fentanyl. Femoral sheaths were removed within three hours post procedure upon a decrease of intravenously administered heparin and groin compressions were applied. Patients were discharged the day after the ablation.

\section{Follow up}

Patients were followed during regular visits at one, six and 12 month(s) after the index procedure in the outpatient service for cardiac arrhythmias. Evaluation of prior 24-h holter monitoring, 12-lead ECG recordings, and arrhythmia episodes were documented. If considered necessary, patients were provided with an episodic ECG portable heart scan (Omron Healthcare Co. Ltd., Kyoto, Japan) for a period of 14 days and the results were analyzed in an extra visit. All data were entered into the hospital information system, which served as source documentation for our analysis.

\section{Radiation dose assessment}

A total radiation dose was calculated for both the ablation procedure and the left atrial imaging (3DRA or CT). Regarding the different use of units, we compared the effective radiation dose $(\mathrm{mSv})$. The amount of radiation during cardiac CT scan was expressed as „dose length product“ ( $\left.\mathrm{mGy} \mathrm{cm}^{1}\right)$ and was adjusted using a conversion coefficient $0,017 \mathrm{mSV} \mathrm{mGy}^{-1} \mathrm{~cm}^{-1}\left(\right.$ ref. $\left.^{21}\right)$. Radiation exposure for 3D rotational angiography was measured as „dose area product“ $\left(\mathrm{mGy}^{2} \mathrm{~cm}^{2}\right)$ and the ef- fective dose was calculated using a conversion coefficient $0,18 \mathrm{mSVmGy}^{-1} \mathrm{~cm}^{-2}\left(\right.$ ref. $\left.^{22}\right)$.

\section{Statistical analysis}

Patient characteristics and the clinical outcome of both groups were described using descriptive statistics. Continuous variables are expressed as a mean \pm standard deviation (SD) and categorical variables as absolute values and percentages. All parameters were compared according to the imaging method used. Categorical data were analyzed using chi-squared test or Fisher's exact test. Continuous data were evaluated using unpaired Student's t-test or nonparametric Mann-Whitney when dealing with non-normal distribution. Results were deemed statistically significant for a $P$ value $>0.5$ since all analyses were performed at the $5 \%$ significance level.

\section{RESULTS}

The study sample involved 125 patients with paroxysmal or persistent atrial fibrillation who received therapeutic catheter ablation at the Arrhythmology Department of St. Anne's University Hospital in Brno between January 2011 and August 2012. The ablation procedure was guided either by preoperational CT scan (62 patients) or periprocedural 3D rotational angiography (63 patients). There was no difference in age, gender, BMI, left atrium diameter, left ventricular ejection fraction, concomitant diseases or proportion of paroxysmal to persistent $\mathrm{AF}$ between both groups. The overall characteristics are presented in Table 1.

For rotational angiography, only direct (left atrial) contrast injection approach was applied with a success rate of $98.5 \%$. The $3 \mathrm{D}$ reconstruction was unsuccessful in one patient due to large LA diameter and poor isocentering associated with incomplete resulting image (pulmo- 
Table 1. Patients' characteristics.

\begin{tabular}{lccc}
\hline & CT (N=62) & DRA (N=63) & \\
\hline Parameter & $\mathrm{N}(\%)$ & $\mathrm{N}(\%)$ & $P^{*}$ \\
\hline Gender (male) & $44(71 \%)$ & $48(76 \%)$ & 0.508 \\
Hypertension & $43(69 \%)$ & $42(67 \%)$ & 0.747 \\
Diabetes mellitus & $14(23 \%)$ & $9(14 \%)$ & 0.231 \\
Hyperlipoproteinemia & $32(52 \%)$ & $29(46 \%)$ & 0.533 \\
Ischemic heart disease & $11(18 \%)$ & $6(10 \%)$ & 0.180 \\
Stroke & $4(6 \%)$ & $2(3 \%)$ & 0.392 \\
Type of atrial fibrillation: & & & 0.480 \\
Paroxysmal & $44(71 \%)$ & $41(65 \%)$ & \\
Persistent & $18(29 \%)$ & $22(35 \%)$ & \\
\hline & Mean (SD) & Mean (SD) & \\
\hline Age (years) & $60.3(10.28)$ & $58.3(9.61)$ & 0.215 \\
Left atrial diameter (mm) & $43.2(6.51)$ & $45.5(6.27)$ & 0.082 \\
Left ventricular ejection & $56.5(9.55)$ & $57.2(7.02)$ & 0.888 \\
fraction (\%) & & & \\
BMI & $29.5(5.1)$ & $29.3(4.0)$ & 0.728 \\
\hline
\end{tabular}

nary vein ostia were not depicted). Conversely, the CT scan yielded $100 \%$ success profile.

There was a close correlation in overall procedural parameters between both groups. No difference was observed in procedural time $(231 \pm 38$ vs. $249 \pm 57 \mathrm{~min}$, $P=0.094)$, fluoroscopic time ( $24.6 \pm 8$ vs. $24 \pm 7.5 \mathrm{~min}$, $P=0.702$ ) or number of RF applications ( 78.21 vs. $84 \pm 23$,

Table 2. Procedure results.

\begin{tabular}{lccc}
\hline & $\begin{array}{c}\mathrm{CT} \\
(\mathrm{N}=62)\end{array}$ & $\begin{array}{c}\text { 3DRA } \\
(\mathrm{N}=63)\end{array}$ & \\
\hline $\begin{array}{l}\text { Parameters } \\
\text { of procedure }\end{array}$ & $\begin{array}{c}\text { Mean } \\
(\mathrm{SD})\end{array}$ & $\begin{array}{c}\text { Mean } \\
(\mathrm{SD})\end{array}$ & $P^{*}$ \\
\hline $\begin{array}{l}\text { Procedural time } \\
\text { (minutes) }\end{array}$ & 231.6 & 249.5 & 0.094 \\
$\begin{array}{l}\text { Fluoroscopic time } \\
\text { (minutes) }\end{array}$ & $(38.64)$ & $(57.68)$ & \\
$\begin{array}{l}\text { Number of RF } \\
\text { applications }\end{array}$ & $(7.99)$ & 24.0 & 0.702 \\
$\begin{array}{l}\text { Ablation time } \\
\text { (seconds) }\end{array}$ & $(21.23)$ & $(7.46)$ & \\
$\begin{array}{l}\text { Radiation exposure } \\
\text { (mSv) }\end{array}$ & 2732.2 & 84.2 & 0.174 \\
\hline
\end{tabular}

$P=0.174)$. However, ablation time ( $2732 \pm 748$ vs. $3105 \pm$ $850, P=0.014)$ and radiation exposure (17.66 \pm 2.32 vs. $\left.5.99 \pm 2.68 \mathrm{mGycm}^{2}, P<0.01\right)$ were significantly lower for 3DRA-guided procedures (Table 2). The mean follow-up period was $13.8 \pm 3.5$ months. The frequency of recurrent episodes of AF (24\% in CT-guided group vs. $27 \%$ in 3DRA-guided group, $P=0.721$ ) as well as the onset of atypical atrial flutter after the index procedure (10\% vs. $8 \%$, respectively, $P=0.731$ ) were similar in both groups (Table 3). No difference in the number of patients necessitating repeat ablation ( $5 \%$ vs. $5 \%, P=0.984$ ) was observed. During the follow-up period, both groups showed a corresponding proportion of prescribed antiarrhythmic drugs ( $55 \%$ vs. $49 \%, P=0.529$ ) and present anticoagulation therapy ( $42 \%$ vs. $46 \%, P=0.777$ ). Procedural complications of ablations guided by 3DRA were comparable with

Table 3. Clinical results.

\begin{tabular}{|c|c|c|c|}
\hline & $\begin{array}{c}\mathrm{CT} \\
(\mathrm{N}=62)\end{array}$ & $\begin{array}{c}\text { 3DRA } \\
(\mathrm{N}=63)\end{array}$ & \\
\hline One-year follow-up & N (\%) & $\mathrm{N}(\%)$ & $P^{*}$ \\
\hline $\begin{array}{l}\text { Recurrent of atrial } \\
\text { fibrillation }\end{array}$ & $15(24 \%)$ & $17(27 \%)$ & 0.721 \\
\hline $\begin{array}{l}\text { Onset of atypical atrial } \\
\text { flutter }\end{array}$ & $6(10 \%)$ & $5(8 \%)$ & 0.731 \\
\hline Antiarrhythmic drugs & $34(55 \%)$ & $31(49 \%)$ & 0.529 \\
\hline Anticoagulation therapy & $26(42 \%)$ & $28(44 \%)$ & 0.777 \\
\hline Repeat ablation & $3(5 \%)$ & $3(5 \%)$ & 0.984 \\
\hline $\begin{array}{l}\text { Procedural complications } \\
\text { (hemodynamically } \\
\text { insignificant pericardial } \\
\text { effusions) }\end{array}$ & $1(2 \%)$ & $3(5 \%)$ & 0.568 \\
\hline
\end{tabular}

those guided by CT ( $2 \%$ vs. $3 \%$, respectively, $P=0.568$ ). Only hemodynamically insignificant pericardial effusions not requiring any intervention were reported. No other complications were encountered.

\section{DISCUSSION}

Prior studies have already shown that 3DRA reconstruction of left atrium is a feasible, simple and safe method with a high rate of success. The main benefit of the approach is online image acquisition during the electrophysiological procedure at the cath lab and the ability to overlay resulting $3 \mathrm{D}$ shell onto the live fluoroscopic screen.

The 3DRA data has proven equal to a cardiac CT scan with no difference in terms of left atrial parameters, such as PV ostia or LA volume measurements. Additionally, patients are subjected to a lower dose of radiation and contrast medium compared to CT. More recently, studies investigating the utility of 3DRA for imaging the right or left ventricle as guidance for catheter ablation of ventricular arrhythmias have been published ${ }^{23}$.

To the best of our knowledge, there are only limited research data available assessing clinical outcome of $\mathrm{AF}$ ablation guided by 3DRA vs. CT. A study conducted by Knecht et al. is one of the few examples addressing the topic ${ }^{24}$. In a randomized trial, they compared clinical outcome of catheter ablation for atrial fibrillation supported by conventional electroanatomical mapping using the CARTO system with procedures guided by $3 \mathrm{D}$ rotational angiography. Between 2007 and 2008, they assigned 91 patients with paroxysmal (63\%) and persistent (37\%) atrial fibrillation referred for ablation in Boston and Bordeaux. Those patients were randomized to either CARTO-guided (47 patients) or 3DRA-guided (44 patients) ablation. The data showed close correlation in procedural time $(232 \pm$ 65 vs. $218 \pm 67 \mathrm{~min}, P=0.335$ ), fluoroscopy time ( $75 \pm$ 
28 vs. $67 \pm 26 \min , P=0.151)$, or radiation dose $(71810 \pm$ 42954 vs. $\left.68009 \pm 38345 \mathrm{mGycm}^{2}, P=0.719\right)$ between the groups. Patients were followed for a mean period of 10 \pm 4 months and no difference in AF recurrence (20\% vs. $15 \%, P=0.555)$ or any episodes of recurrent arrhythmia ( $34 \%$ vs. $38 \%, P=0.668$ ) were observed.

Our results are in concordance with these findings. Only procedural and ablation time were non-significantly longer in the 3DRA-guided group and could be explained by extra time needed for the performance of LA rotational angiography which has been a more aggressive approach in the management of atrial fibrillation at our center during the last two years associated with longer duration of RF delivery. We also found a statistically significant reduction in radiation exposure in those procedures supported by 3DRA compared to CT. Based on our experience, a merger of 3DRA with live fluoroscopy enables us to apply a lower X-ray dose in order to produce the same image sequence which can potentially decrease the radiation exposure in procedures while reaching the same fluoroscopic time. The frequency of AF recurrence was $24 \%$ in 3DRA-guided and $27 \%$ in the CT-guided group ( $P=0.721)$ and recurrent arrhythmia in general was $34 \%$ vs. $35 \%$, $P=0.902$. The proportion of arrhythmia-free patients in our sample was slightly lower than Knecht et al., but the mean follow-up period was on average four months longer (13.8 \pm 3.5 to $10 \pm 4$ months), possibly leading to a higher occurrence of arrhythmia episodes. The high safety profile of 3D rotational angiography was achieved in both study samples (ours vs. Knecht) with no difference in procedural risks compared to conventional procedures.

Another study investigating clinical outcomes of procedures employing 3D rotational angiography was performed by Carpen et al. ${ }^{12}$. This retrospective analysis confirmed that fusion of 3DRA with the electroanatomical mapping system results in reduction of total procedural time and radiation exposure with similar clinical outcome during the follow-up ( $10 \pm 3$ months vs. $11.9 \pm 5.3$ months) compared to procedures without the fusion. Despite the small sample size (36 patients), this conclusion supports the application of 3DRA to guide ablations for atrial fibrillation.

These findings imply a clinical benefit of 3D rotational angiography employed in routine practice. Knecht et al. used 3DRA as a stand-alone imaging method while we dispute 3DRA as an adequate substitute for the conventional electroanatomical mapping since specific functionalities, such as voltage and activation mapping, are not supported and can be useful in patients converting from AF into another atrial arrhythmia (e. g. atypical atrial flutter) during the procedure. Interventions guided only by 3DRA could also be challenging for less experienced physicians.

However, retrospective analysis and a shorter followup period are the main limitations of our study. Another limitation is consecutive inclusion of patients and potential bias of results with the growing experience of operators performing the AF ablation. In contrast, our ablation technique was constant during this period. The sample size in our study was larger than both the retrospective and prospective trials reporting on clinical outcomes of
AF ablation using 3DRA (ref. ${ }^{12,24}$ ). Further confirmation of the retrospective data with a prospective, randomized study is required, but is not ethically justified due to the superiority of 3DRA in reducing radiation exposure documented in a number of studies ${ }^{6,7,10}$. On the other hand, a new state-of-the-art cardiac CT often subjects patients to a lower dose of radiation with values close to the $3 \mathrm{D}$ rotational angiography ${ }^{25}$.

Based on the literature, no prior study has compared the clinical outcomes of ablation procedures using 3D rotational angiography as a substitute to cardiac CT. Both Tang et al. and Kriatselis et al. reported a reduction in the dose of radiation and contrast medium for patients undergoing 3DRA in comparison to CT. Our clinical results demonstrate the utility of $3 \mathrm{D}$ rotation angiography as an adequate alternative to a commonly performed CT scan ${ }^{6,7}$.

\section{CONCLUSION}

Three-dimensional rotational angiography has proven to be a safe and simple method for imaging the left atrium and guiding catheter ablation for atrial fibrillation. Our clinical experience suggests that 3DRA is comparable to a conventional CT scan. Given the low radiation exposure and use of contrast medium in comparison to CT, this approach could be a useful alternative technique in $3 \mathrm{D}$ reconstruction of the left atrium as a support for $\mathrm{AF}$ ablation.

\section{ABBREVIATIONS}

3D, three dimensional; 3DRA, three-dimensional rotational angiogram; AF, atrial fibrillation; CT, computed tomography; MRI, magnetic resonance imaging.

Acknowledgement: Supported by European Regional Development Fund, Project FNUSA-ICRC (No. CZ 1.05/1.1.00/02.0123)

Author contributions: FL, ZS: study design; FL, JJ, JW, TK, AZ, RJ: data collection; FL, ZS, MN: data interpretation; FL, RS, TK: statistical analysis; FL: manuscript writing; FL, JW: figures; ZS, MN, PK, JV: final approval. Conflict of interest statement: The authors state that there are no conflicts of interest regarding the publication of this article.

\section{REFERENCES}

1. Anderson JL., Halperin JL, Albert NM, Bozkurt B, Brindis RG, Curtis LH, DeMets D. Management of Patients With Atrial Fibrillation (Compilation of 2006 ACCF/AHA/ESC and 2011 ACCF/AHA/HRS Recommendations) A Report of the American College of Cardiology/ American Heart Association Task Force on Practice Guidelines. Circulation 2013;127(18):1916-26.

2. Schwartzman D, Lacomis J, Wigginton WG. Characterization of left atrium and distal pulmonary vein morphology using multidimensional computed tomography. J Am Coll Cardiol 2003;41(8):1349-57.

3. Dong J, Dickfeld T, Dalal D, Cheema A, Vasamreddy CR, Henrikson CA, Marine JE. Initial experience in the use of integrated electro- 
anatomic mapping with three-dimensional MR/CT images to guide catheter ablation of atrial fibrillation. J Cardiovasc Electrophysiol 2006;17(5):459-66.

4. Martinek M, Nesser H-J, Aichinger J, Boehm G, Purerfellner H. Impact of integration of multislice computed tomography imaging into three-dimensional electroanatomic mapping on clinical outcomes, safety, and efficacy using radiofrequency ablation for atrial fibrillation. Pacing Clin Electrophysiol PACE 2007;30(10):1215-23.

5. Malchano ZJ, Neuzil P, Cury RC, Holmvang G, Weichet J, Schmidt EJ, Ruskin JN, Reddy VY. Integration of cardiac CT/MR imaging with three-dimensional electroanatomical mapping to guide catheter manipulation in the left atrium: implications for catheter ablation of atrial fibrillation. J Cardiovasc Electrophysiol 2006;17(11):1221-9.

6. Tang M, Kriatselis C, Ye G, Nedios S, Roser M, Solowjowa N, Fleck $\mathrm{E}$, Gerds-Li J-H. Reconstructing and registering three-dimensional rotational angiogram of left atrium during ablation of atrial fibrillation. Pacing Clin Electrophysiol PACE 2009;32(11):1407-16.

7. Kriatselis C, Nedios S, Akrivakis S, Tang M, Roser M, Gerds-Li J-H Fleck E, Orlov MV. Intraprocedural imaging of left atrium and pulmonary veins: a comparison study between rotational angiography and cardiac computed tomography. Pacing Clin Electrophysiol PACE 2011;34(3):315-22.

8. Dong J, Calkins H, Solomon SB, Lai S, Dalal D, Lardo A, Brem E. Integrated Electroanatomic Mapping With Three-Dimensional Computed Tomographic Images for Real-Time Guided Ablations. Circulation 2006;113(2):186-94.

9. Earley MaJ, Showkathali R, Alzetani M, Kistler PM, Gupta D, Abrams DJ, Horrocks JA, Harris SJ, Sporton SC, Schilling RJ. Radiofrequency ablation of arrhythmias guided by non-fluoroscopic catheter location: a prospective randomized trial. Eur Heart J 2006;27(10):1223-9.

10. Lehar F, Starek Z, Jez J, Novak M, Wolf J, Kruzliak P, Kulik T, Zbankova A. Rotational atriography of left atrium - a new imaging technique used to support left atrial radiofrequency ablation: a comparison of anatomical data of left atrium. Interv Akut Kardiol 2013;12(4):184-9.

11. Li JH, Haim M, Movassaghi B, Mendel JB, Chaudhry GM, Haffajee Cl, Orlov MV. Segmentation and registration of three-dimensional rotational angiogram on live fluoroscopy to guide atrial fibrillation ablation: a new online imaging tool. Heart Rhythm Off J Heart Rhythm Soc 2009;6(2):231-7.

12. Carpen M, Matkins J, Syros G, Gorev MV, Alikhani Z, Wylie JV, Natan SR. First experience of 3D rotational angiography fusion with NavX electroanatomical mapping to guide catheter ablation of atrial fibrillation. Heart Rhythm Off J Heart Rhythm Soc 2013;10(3):422-7.

13. Bertaglia E, Bella PD, Tondo C, Proclemer A, Bottoni N, Ponti RD, Landolina M. Image integration increases efficacy of paroxysmal atrial fibrillation catheter ablation: results from the CartoMerge Italian Registry. Eur Eur Pacing Arrhythm Card Electrophysiol J Work Groups Card Pacing Arrhythm Card Cell Electrophysiol Eur Soc Cardiol 2009;11(8):1004-10.

14. Caponi D, Corleto A, Scaglione M, Blandino A, Biasco L, Cristoforetti Y, Cerrato N, Toso E, Morello M, Gaita F. Ablation of atrial fibrilla- tion: does the addition of three-dimensional magnetic resonance imaging of the left atrium to electroanatomic mapping improve the clinical outcome?: a randomized comparison of Carto-Merge vs. Carto-XP three-dimensional mapping ablation in patients with paroxysmal and persistent atrial fibrillation. Eur Eur Pacing Arrhythm Card Electrophysiol J Work Groups Card Pacing Arrhythm Card Cell Electrophysiol Eur Soc Cardiol 2010;12(8):1098-104.

15. Nölker G, Gutleben KJ, Asbach A, Vogt J, Heintze J, Brachmann J, Horstkotte D, Sinha AM. Intracardiac echocardiography for registration of rotational angiography-based left atrial reconstructions: a novel approach integrating two intraprocedural three-dimensional imaging techniques in atrial fibrillation ablation. Eur Eur Pacing Arrhythm Card Electrophysiol J Work Groups Card Pacing Arrhythm Card Cell Electrophysiol Eur Soc Cardiol 2011;13(4):492-8.

16. Thiagalingam A, Manzke R, D'avila A, Ho I, Locke AH, Ruskin JN, Chan RC, Reddy VY. Intraprocedural Volume Imaging of the Left Atrium and Pulmonary Veins with Rotational X-Ray Angiography: Implications for Catheter Ablation of Atrial Fibrillation. J Cardiovasc Electrophysiol 2008;19(3):293-300.

17. Orlov MV. How to perform and interpret rotational angiography in the electrophysiology laboratory. Heart Rhythm 2009;6(12):1830-6.

18. Gage BF, Waterman AD, Shannon W, Boechler M, Rich MW, Radford MJ. Validation of clinical classification schemes for predicting stroke: Results from the national registry of atrial fibrillation. JAMA 2001;285(22):2864-70.

19. Ector J, Buck SD, Nuyens D, Rossenbacker T, Huybrechts W, Gopal $R$, Maes $F$, Heidbüchel $H$. Adenosine-induced ventricular asystole or rapid ventricular pacing to enhance three-dimensional rotational imaging during cardiac ablation procedures. Europace 2009;11(6):751-62.

20. Orlov MV, Hoffmeister P, Chaudhry GM, Almasry I, Gijsbers HM, Swack T, Haffajee $\mathrm{Cl}$. Three-dimensional rotational angiography of the left atrium and esophagus-A virtual computed tomography scan in the electrophysiology lab? Heart Rhythm 2007;4(1):37-43.

21. Einstein AJ, Moser KW, Thompson RC, Cerqueira MD, Henzlova MJ. Radiation dose to patients from cardiac diagnostic imaging. Circulation 2007;116(11):1290-1305.

22. Schultz FW, Zoetelief J. Dose conversion coefficients for interventional procedures. Radiat Prot Dosimetry 2005;117(1-3):225-30.

23. Orlov MV, Ansari MM, Akrivakis ST, Jadidi A, Nijhof N, Natan SR, Wylie JV, Hicks A, Armstrong J, Jais P. First experience with rotational angiography of the right ventricle to guide ventricular tachycardia ablation. Heart Rhythm 2011;8(2):207-11.

24. Knecht S, Wright M, Akrivakis S, Nault I, Matsuo S, Chaudhry GM, Haffajee $C$. Prospective randomized comparison between the conventional electroanatomical system and three-dimensional rotational angiography during catheter ablation for atrial fibrillation. Heart Rhythm 2010;7(4):459-65.

25. Yang L, Xu L, Yan Z, Yu W, Fan Z, Lv Z, Zhang Z. Low dose 320-row CT for left atrium and pulmonary veins imaging-the feasibility study. Eur J Radiol 2012;81(7):1549-54. 